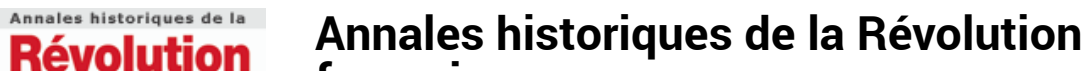 \\ française française
}

340 | avril-juin 2005

Les arts et la révolution

\section{Études et notaires parisiens en 1803, au moment de la loi du 25 ventôse an XI (16 mars 1803)}

\section{Émile Ducoudray}

\section{(2) OpenEdition \\ 1 Journals}

\section{Édition électronique}

URL : https://journals.openedition.org/ahrf/2027

DOI : 10.4000/ahrf.2027

ISSN : 1952-403X

Éditeur :

Armand Colin, Société des études robespierristes

\section{Édition imprimée}

Date de publication : 1 juin 2005

Pagination : 201-205

ISSN : 0003-4436

\section{Référence électronique}

Émile Ducoudray, «Études et notaires parisiens en 1803, au moment de la loi du 25 ventôse an XI (16 mars 1803) ", Annales historiques de la Révolution française [En ligne], 340 | avril-juin 2005, mis en ligne le 20 avril 2006, consulté le 23 avril 2022. URL : http://journals.openedition.org/ahrf/2027 ; DOI :

https://doi.org/10.4000/ahrf.2027

\section{Ce document a été généré automatiquement le 23 avril 2022}

Tous droits réservés 


\title{
Études et notaires parisiens en 1803, au moment de la loi du 25 ventôse an XI (16 mars 1803)
}

\author{
Émile Ducoudray
}

\section{RÉFÉRENCE}

Philippe Bertholet, Études et notaires parisiens en 1803, au moment de la loi du 25 ventôse an XI (16 mars 1803) Paris, Éd. Association des notaires du Châtelet, 2004, 663 p., $27.50 €$.

1 Voici un ouvrage qui fera date pour tous ceux qui s'intéressent à l'histoire économique et sociale de la capitale. Le notariat parisien est mal connu. Sans doute disposons-nous de travaux sur sa pratique dans plusieurs domaines, comme par exemple la thèse de Jacques Lelièvre sur La pratique des contrats de mariage chez les notaires du Châtelet de Paris de 1769 à 1804 (1959), sans oublier les grandes enquêtes sur les contrats de mariage sous l'Ancien Régime (R. Mousnier), les testaments (P. Chaunu), les inventaires après décès (D. Roche, A. Pardailhé-Galabrun), le crédit privé (PT. Hoffmann, G. Postel-Vinay, J.-L. Rosenthal). Mais les études institutionnelles et sociales sur le milieu notarial lui-même, son activité (le nombre des actes par étude), son rôle dans la société parisienne ne sont pas nombreuses. Nous avons certes, le solide travail de Marie-France Limon sur Les notaires du Châtelet de Paris sous le règne de Louis XIV (1992), mais pour la période qui va de 1715 au Consulat - si l'on excepte l'ouvrage classique mais un peu ancien de F. Foiret Une corporation parisienne pendant la Révolution, les notaires (1912) - il n'y a pas grand chose, sinon quelques articles, par définition limités. D'où l'importance du travail de Ph. Bertholet.

2 On sait que la Révolution supprima les corporations. Par conséquent, les compagnies de notaires n'eurent plus d'existence légale: plus de bureaux, plus de réunions, plus d'assemblées. Après le coup d'État de Brumaire (novembre 1799), la compagnie de la capitale, après neuf années d'inorganisation de la profession, commença à se 
reconstituer. Elle reprit ses activités, ses traditions, assura de nouveau la transmission des charges et présenta au préfet les nouveaux notaires. Mais c'est la loi du 25 ventôse an XI qui réorganisa le notariat. Elle n'accorda pas au notariat parisien, comme sous l'Ancien Régime, l'extension de sa compétence à tout le territoire de la France, mais elle céda sur le nombre des notaires de la capitale. La loi de 1803 décidait en effet que pour les villes de 100000 habitants et au-dessus, il y aurait au maximum un notaire pour 6 000 habitants. Or, avec la diminution de la population attestée par les dénombrements de l'an V (1797) et de l'an IX (1801), le nombre des notaires n'aurait pas dû excéder 100. Mais, les notaires parisiens insistèrent vivement pour que leur nombre ne fût pas diminué et le ministre de la Justice fut d'avis de conserver les 114 notaires du début de la Révolution : les 113 notaires du Châtelet, un nombre qui remontait au règne de Henri IV auxquels s'ajoutait, depuis 1790, celui du Roule rattaché à la capitale.

3 Ce sont ces 114 notaires de 1803 qui sont l'objet de l'étude socio-professionnelle magistrale de Ph. Bertholet. L'origine géographique de ces notaires est sans surprise. Elle ne diffère guère de l'ensemble de la population parisienne. La moitié d'entre eux était née dans la capitale et appartenait majoritairement à des familles établies à Paris depuis au moins deux générations. Les autres venaient de petites villes proches de la capitale ou de provinces situées au nord de la Loire. En revanche, leur origine sociale est plus originale. La charge notariale parisienne, à cette date, se transmet rarement de père en fils. Deux notaires seulement étaient fils de confrères parisiens et cinq autres fils de notaires royaux de province. Les notaires de 1803 ne sont donc pas issus de la profession. Leur immense majorité vient du milieu social du négoce, de la marchandise, de l'entreprise ou de la fabrique (50\%), de la judicature (près de $20 \%$ ) ou des administrations de l'Ancien Régime (13\%). Une situation bien différente de celle du Premier Empire, comme l'a montré Nicole Célestin (1970), où $38 \%$ des notaires proviennent du milieu notarial, $28 \%$ de celui de la judicature et seulement $21 \% \mathrm{du}$ monde du négoce et de la marchandise. L'année 1803 est bien une phase de transition et de mutation sociale.

4 Leur formation est essentiellement pratique. Si, pour 15 futurs notaires de 1803, elle se complète par une formation juridique acquise à la Faculté de droit, c'est l'apprentissage qui est la règle générale. Le temps de cléricature varie de 10 à 30 ans. Ainsi, Charles Lemaire, fils d'un marchand épicier et cirier de Nanteuil-le-Haudoin, entre à l'âge de 15 ans en 1758, comme petit clerc dans l'étude de Pierre Lecousturier où il passera 29 ans. En 1787, à l'âge de 44 ans, il achètera l'office et la pratique du notaire Pierre Paulnier, dans le quartier du Panthéon, moyennant 290000 livres. Il exercera pendant 18 ans jusqu'en 1805. Dans l'ensemble, ces notaires étaient entrés en fonction à l'âge de 33 ans et en mars 1803, leur âge moyen était de 45 ans et demi. À cette date, par leur recrutement, les 114 notaires ne formaient pas un groupe homogène. Ph. Bertholet distingue trois strates. Les 45 titulaires d'un office notarial au Châtelet de Paris, devenus « notaires publics » pendant la Révolution, avaient acheté leur charge sous l'Ancien Régime à un prix très élevé, environ 290000 livres, pour la période 1787-1790. Ils avaient reçu leur habilitation après avoir prêté serment devant le doyen et les représentants de la compagnie des notaires de Paris. Venaient ensuite les 21 notaires d'institution républicaine, ceux qui avaient été reçus au concours de l'an II. Ici, il faut noter une erreur surprenante sous la plume de Ph. Bertholet. Ce concours n'avait pas eu lieu le 25 thermidor an II - 12 août 1794, mais s'était déroulé du 2 ventôse (20 février) au 29 germinal (18 avril 1794). Il était destiné à remplacer les notaires destitués, démissionnaires ou décédés. Ces nouveaux venus négocièrent avec les héritiers de leurs 
prédécesseurs ou avec le prédécesseur lui-même le prix de la pratique et des recouvrements de l'étude, mais dans une conjoncture financière avantageuse liée à la dévaluation de l'assignat. On trouvait enfin les 48 notaires nommés sous le Directoire et le Consulat par arrêté du département et qui avaient prêté serment devant le Tribunal de première instance de la Seine. Sans doute avaient-ils payé leur charge en valeur numéraire, beaucoup plus cher que celles des notaires « carmagnoles» de l'an II, mais sans atteindre pour autant les sommes de l'Ancien Régime. Entre 1797 et 1800, les charges notariales se négocient entre 20000 et 46000 francs. Mais les revenus étaient confortables: autour de 30000 francs par an sous le Consulat et l'Empire, voire 40000 francs pour les études les plus achalandées.

5 Si 10 notaires de 1803 vivaient maritalement sans avoir contracté d'alliances, la grande majorité avait fait le choix du mariage. L'âge moyen au premier mariage est d'environ 35 ans. 17 notaires s'étaient déjà mariés avant d'entrer en fonction. C'est un pourcentage nettement supérieur à celui des notaires du XVIIe siècle étudiés par

M.-F. Limon : elle n'a trouvé, dans ce cas, qu'un seul notaire sur un échantillon de 82 . Une explication plausible est fournie par Ph. Bertholet. Les deux-tiers des notaires de 1803 avaient été nommés sous la Révolution et le Consulat à un moment où le prix des charges était très inférieur à celui de l'Ancien Régime. L'apport de la dot de la future épouse était donc moins nécessaire pour le remboursement de tout ou partie du prix de l'office. Les épouses, parisiennes dans leur majorité, étaient beaucoup plus jeunes que leurs maris. Leur âge moyen atteint les 22 ans. Il faut aussi remarquer que le choix des alliances matrimoniales ne passe pas automatiquement par l'endogamie au sein de la profession. 18 notaires sur 104 mariés avaient choisi leurs épouses dans le milieu d'un confrère parisien et 11 seulement avaient épousé la fille d'un notaire. Les autres s'étaient mariés dans le milieu social dont ils étaient issus. Abordant la hiérarchie des fortunes notariales en 1803, on peut regretter que, par un excès de scrupules, $\mathrm{Ph}$. Bertholet n'ait pas voulu établir le tableau comparé des patrimoines au mariage et au décès. Il estime, en effet, qu'au moment du mariage et à partir de la Révolution, les biens du futur époux, à la différence de la dot de la fille, se ramenaient souvent à une évaluation à l'amiable acceptée par les deux parties. Il n'empêche que cette évaluation ne devait pas être bien loin de la réalité, les familles bourgeoises ne s'en laissant point facilement conter.

6 En revanche, l'enquête de $\mathrm{Ph}$. Bertholet sur les fortunes notariales au décès est exemplaire. Un travail minutieux dans les tables de succession des Archives de Paris et dans les actes du minutier central des Archives nationales lui a permis de retrouver les patrimoines en fin de vie de 87 notaires (les trois-quarts de l'effectif de 1803). C'est un exploit pour quiconque a tâté de ce type d'archives. Ph. Bertholet distingue trois groupes. Viennent en tête 11 notaires possédant au moins un million de francs à leur décès. Certains s'étaient spécialisés dans un domaine particulier: Gauldrée-Boileau était ainsi directeur général des recouvrements des messageries; Lherbette, trésorier de la caisse des placements en viager; Mathieu de Heidolsheim, fournisseur aux armées. Un second groupe, le plus important, comprend 60 notaires dont près des deux-tiers laissaient une fortune comprise entre 200000 et plus de 500000 francs. Enfin, 16 notaires terminaient leur existence avec une succession déficitaire, moins de 10000 francs. Certains avaient fait faillite (parfois, comme Faucompret, pour avoir été trop accommodant et s'être montré incapable de refuser un prêt à ses clients), d'autres 
avaient fait de mauvaises affaires et quelques-uns, comme Lefebvre de Saint-Maur ou Raguideau, le notaire de l'Empereur, pour avoir vécu au-dessus de leurs moyens.

7 Les notaires de 1803 jouèrent un rôle non négligeable dans la société de leur époque. Ils ne furent véritablement exclus de la vie publique parisienne qu'entre la chute du trône et celle de Robespierre. Sauf en 1792, on les retrouve dans toutes les assemblées électorales de Paris, de 1789 à 1799. Lors des élections départementales de l'an IX (1801), l'ensemble des notaires de Paris fut inscrit sur la liste des notabilités du département de la Seine. Par la suite, beaucoup furent maires ou adjoints au maire d'un des 12 arrondissements de la capitale, conseillers généraux du département de la Seine. $\mathrm{Ph}$. Bertholet consacre un développement à l'attitude des notaires à l'heure de la mort. Curieusement, chez ces professionnels, un peu plus de la moitié de l'effectif de 1803 (58) rédigea un testament, généralement olographe. Les autres moururent $a b$ intestat, mais on ne peut guère en tirer de conclusion. C'est exactement la même proportion qu'a trouvée M.-F. Limon pour ses notaires au Châtelet, sous le règne de Louis XIV. Il faut remarquer néanmoins que ceux qui avaient été nommés sous l'Ancien Régime (45) ont testé davantage que ceux qui le furent sous la Révolution et le Consulat. 14 de ces testaments (le quart des testataires) témoignent d'un attachement à la religion catholique (préambule religieux, nombre de messes à célébrer pour l'âme du défunt, dons à la paroisse) et 9 font preuve d'une grande dévotion. Un notaire, Turrel, décédé en 1818 à l'âge de 63 ans, abjura la religion catholique, souhaitant être enterré selon le rite protestant.

8 L'examen de l'activité notariale parisienne, à la charnière du XVIIIe et du XIXe siècle, mérite une attention toute particulière. À partir de documents conservés à la Chambre des notaires de la place du Châtelet, Ph. Bertholet a dressé un «tableau des actes délivrés en minutes par 17 études notariales de 1784 à $1820 \%$. Il en tire plusieurs constatations. L'activité notariale reste soutenue jusqu'en 1795. Cette année-là, les notaires parisiens délivrent autant d'actes que dans la période 1784-1788. L'année 1792 fut même exceptionnelle avec une production estimée à 120086 minutes. Or une telle activité est paradoxale dans le contexte révolutionnaire qui, normalement, aurait dû réduire le volume des transactions. Ph. Bertholet rappelle, en effet, que dès 1791, les notaires parisiens avaient perdu des avantages considérables : le droit d'instrumenter dans tout le royaume, l'exemption du contrôle qui attirait à Paris une clientèle provinciale dont les actes étaient soumis à des taxes considérables, le privilège de délivrer auprès du public les emprunts d'État, du clergé, des pays d'États et des compagnies de finances dont ils tiraient de substantiels bénéfices. Ils furent également privés des revenus de l'Église, en particulier des contrats de constitution de rentes passés par l'intermédiaire des agents généraux du clergé. Ils perdirent aussi une riche clientèle émigrée et celle des colons de Saint-Domingue. Il faut ajouter à cela, pendant la Terreur, les arrestations (17 notaires de 1803 l'avaient été) qui sont à l'origine du ralentissement ou de la cessation d'activité d'un grand nombre d'études. Enfin, il y avait la concurrence des juges de paix habilités à délivrer des actes relatifs au droit de la famille. Si le notariat réussit à surmonter cette conjoncture très défavorable, estime $\mathrm{Ph}$. Bertholet, c'est qu'il bénéficia du remboursement par l'État d'un nombre considérable d'offices supprimés et aussi de la vente des biens nationaux qui multiplia le nombre des procurations et des baux de location. On peut faire des réserves sur son second argument. L'explosion de la vente des biens nationaux s'est produite sous le Directoire. Or, précisément pendant cette période, elle n'a pas enrayé la baisse de l'activité notariale. Car sous le Directoire, constate Ph. Bertholet, la chute de la 
production d'actes est impressionnante. À partir des années 1795-1796, elle est de 58 \% par rapport à la période précédente. Ph. Bertholet retrouve ici l'hypothèse développée par P. T. Hoffmann, G. Postel-Vinay, J.-L. Rosenthal (2001). En 1797, la dette privée à Paris était devenue négligeable. La plupart des emprunts anciens ayant été remboursés en assignats dévalués, il s'en suivi un effondrement des transactions d'actes de crédit passés devant notaire. L'hyperinflation acheva la catastrophe. C'est ainsi que le notaire Pierre Raffeneau, contraint d'accepter le remboursement de bonnes créances en papier dévalué et dans l'impossibilité d'obliger ses créanciers à la réciproque, fut acculé à une retentissante faillite en août 1799. L'activité notariale n'a pas réussi à redémarrer sous le Directoire. En 1798, elle était inférieure de $50 \%$ à 1795 et de $61 \%$ à 1792 . L'onde de choc fut terrible : 13 notaires donnèrent leur démission. Elle affecta la vie privée de plusieurs notaires dont les épouses demandèrent la séparation de biens pour préserver leur dot en cas de faillite. Un comportement qui contribua d'ailleurs à aggraver la crise. En 1803, le notariat semble s'être remis des effets de l'inflation et des désordres politiques. Son activité se stabilise à la production de 60000 à 65000 minutes par an qui sera d'ailleurs celle de l'Empire et du début de la Restauration. Mais il a perdu la position dominante qui avait été la sienne sous l'Ancien Régime, particulièrement dans le domaine du crédit.

9 Un chapitre original et novateur de cet ouvrage est consacré à la localisation et à la répartition des études notariales dans la capitale. L'enquête de Ph. Bertholet lui a permis d'identifier les maisons, avec leurs numérotations successives de l'Ancien Régime à nos jours, et de déterminer leurs emplacements actuels quand elles n'ont pas été démolies. En 1803, la quasi totalité des études était située dans un périmètre limité par les boulevards. On comptait sur la rive droite 89 études, dans les îles 5 études (4 dans l'île de la Cité et 1 dans l'île Saint-Louis) et sur la rive gauche 20 études. Tout naturellement, les études se concentraient dans les quartiers les plus dynamiques, là où les activités économiques dégageaient des profits importants. Ph. Bertholet distingue quatre secteurs de l'implantation notariale. Le premier, le plus important avec 70 études, correspondait à la moitié occidentale de la rive droite et comprenait les cinq premiers arrondissements anciens, de la rue du Faubourg Saint-Honoré à la rue SaintDenis et du quai de la Mégisserie à la Chaussée d'Antin. Un second secteur, avec 24 études, couvrait, en y comprenant les îles, la moitié orientale de la rive droite, de la rue Saint-Martin au faubourg Saint-Antoine, de la rue Meslay à l'Hôtel-de-Ville (dans le VIIIe arrondissement on trouvait 2 études dans la division de l'Indivisibilité et aucune dans les trois divisions du faubourg Saint-Antoine). Un troisième secteur était constitué par la moitié occidentale de la rive gauche (les Xe et XIe arrondissements) et comprenait 18 des 20 études au sud de la Seine, du Champ de Mars à la rue SaintJacques, du quai Malanquais au jardin du Luxembourg. Le faubourg Saint-Germain en était le pôle majeur. Enfin, le premier secteur, le XIIe arrondissement, de la rue SaintJacques au jardin des Plantes et du quai de la Tournelle à l'hôpital du Val-de-Grâce, ne comprenait que deux études localisées dans la division du Panthéon-Français. Cette implantation s'accompagnait d'une "stratégie de l'emplacement " que Ph. Bertholet analyse avec finesse: recherche des grands axes de circulation, des places et des carrefours, de la proximité de bâtiments publics permettant aux clients de mieux mémoriser le siège de l'étude, de maisons d'encoignure présentant l'avantage de disposer de deux panonceaux. Cette recherche du «bon emplacement » explique qu'en 1803 près de la moitié des maisons notariales avait moins de vingt ans de durée d'implantation et pas plus de cinq ans pour le quart d'entre elles. Les notaires étaient 
rarement propriétaires de leurs maisons (seulement un quart d'entre eux), alors que le prix moyen des demeures notariales pendant la période du Consulat et de l'Empire ne représentait que trois ans de revenus. Mais, il leur suffisait amplement d'avoir à rembourser le prix de leurs charges, de payer leur cautionnement et de sauvegarder ainsi, en cas d'éventualité, leur mobilité.

La partie centrale de l'ouvrage est consacrée aux 114 notices des notaires de 1803. C'est un trésor d'érudition qui ne peut se résumer et qui fait également de ce livre un précieux instrument de travail. Plutôt que de présenter les notaires par ordre alphabétique, Ph. Bertholet a intelligemment adopté une logique spatiale en utilisant une classification des études par arrondissement ancien (un index alphabétique permet d'ailleurs de se reporter au nom de chaque notaire). Toute investigation sociale relative à un personnage de la Révolution ou de l'Empire, dont on ne connaît que l'adresse, implique la consultation préalable, aux Archives de Paris, de l'état civil reconstitué et des tables de déclarations de succession (DQ 8 et $D Q 7$ ) où sont généralement mentionnés les noms des notaires qui ont instrumenté pour le contrat de mariage, le dépôt de testament ou l'inventaire après décès. Mais il y a parfois de bien tristes déconvenues. C'est alors que les notices de $\mathrm{Ph}$. Bertholet présentent un intérêt considérable. Sans doute, les Parisiens ne passaient-ils pas automatiquement des actes dans des études proches de leurs domiciles, mais l'expérience prouve que beaucoup d'entre eux s'adressaient aux notaires de leur quartier ou d'un quartier proche et qu'ils gardaient cette habitude toute leur vie. C'est dans cette perspective que Ph. Bertholet a réalisé une série de 21 plans d'arrondissements avec leurs divisions (ex-sections) à partir d'agrandissements du plan de Maire (1803) avec la localisation minutieuse des études notariales. Comme nombre de ces études étaient déjà installées à la même adresse dès le milieu du XVIIIe siècle et qu'elles le seront encore jusqu'au milieu du XIXe siècle, il est superflu de souligner l'intérêt de ce magnifique travail.

On ne saurait trop remercier la Chambre des notaires de Paris, soucieuse de célébrer le double centenaire de la loi de ventôse en 2003 et du Code civil en 2004, d'avoir eu l'initiative de publier, malgré son volume, cet ouvrage qui laisse bien augurer de la thèse de l'auteur, en préparation, sur les notaires parisiens du début du règne de Louis XVI à la loi de ventôse an XI. 